NOVEMBER 1978

PPPL-1487

UC-20g

MASTER

\title{
STABILITY OF HELICALLY SYMMETRIC EQUILIBRIUM TO IDEAL MHD PERTURBATIONS
}

BY

E, J, VALEO, P, K. KAW,

D. A, MONTICELLO AND R, B, WHITE

\section{PLASMA PHYSICS LABORATORY}

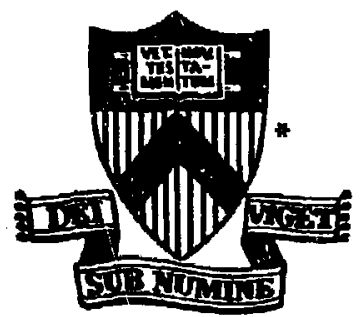

\section{PRINCETON \\ UNIVERSITY PRINCETON, NEW JERSEY}

Th1s work was supported by the $\dot{U}$. $\dot{S}$. Department of Energy Contract No. EY-76-6-02-3073. Reproduction, tranglation, publication, use and disposal, in thole or in part, by or for the Dnited States Government is permitted. 



\section{INTRODUCTION}

There is currently substantial experimental evidence that disruptions in tokamaks are strongly correlated with tearing mode activity at low order rational surfaces. ${ }^{1-4}$ These modes are driven by the free energy associated with the nonuniform current density profile. 5 The modes do not exist in the ideal MHD description because they result in changes in magnetic field topology (reconne:tion); such changes are not allowed in ideal inHD. The linear growth rates are intermediate between the resistive diffuion rate $l / \tau_{R}=c \eta / a^{2}$ and the Alfven transit rate $1 / T_{A}=B_{\theta} / \sqrt{0} a$. Here a is the column radius, $n$ the resistivity, $\rho$ the mass density, and $B_{\theta}$ the poloidal magnetic field strength. The nonlinear state of the collisional tearing mode has been the subject of extensive numerical study. Until recently, these studies have been limited to two diliensional computations; viz., the time development of helically symmetric island states on a cylindrically symmetric equilibrium ${ }^{6-9}$ (the cylinder, with its ends identified, being treated as a model for a small inverse aspect ratio torus). These two-dimensional nonlinear numerical studies (of low B cylindrical columns) have shown the creation of magnetic islands at the mode rational surface where $m-n q=0$. Here $m$ and $n$ are the azimuthal and axial mode numbers respectively and $\mathrm{g}(r) \equiv \mathrm{RB}_{\theta}(\mathrm{r}) / \mathrm{rB}_{\mathrm{Z}}$ is the safety factor. It is just at such a surface that the ideal MHD constraint $\mathrm{E} \cdot \mathrm{B}=\mathrm{O}$ is violated. since these nonlinear states have a 
(helical) symmetry direction, magnetic surfaces are present at all times. This is fundamental to the transport properties of the column. If field lines are confined to the neighborhood of surfaces, transport losses will be determined by the relatively small fluxes perpendicular to these surfaces. Thus the creation of magnetic islands of a single helicity can explain the experimentally observed quenching of the discharge, only for the case where the island width itself becomes comparable to the discharge radius. 10

If, however, these magnetic surfaces are destroyed, then some field lines ergodically $f-11$ a volume and the much larger fluxes parallel to the field line direction can lead to enhanced radial losses ${ }^{11-13}$ and possible disruption of the discharge. Magnetic surface destruction can result from three dimensional effects associated with toroidal coupling terms ${ }^{14-16}$ or excitation of mudes with more than one helicity. 16-13 Recent threedimensional simulations of tearing mode development in a zero $B$ cylindrical column have demonstrated that modes of several helicities can be driven to large amplitudes. ${ }^{18}$ This could lead to a destruction of magnetic surfaces over a substantial fraction of the column radius. These simulations have been interpreted via a heuristic thuory in which linear tearing eigenmodes lacalized at different mode rational surfaces are nonlinearly coupled. 19 The mode-coupling equations are normalized to the simulation results in order to obtain the unknown matrix elements 
and then the results for the nonlinear growth rates are scaled to realistic tokamak parameters.

The generation of such symmetry-breaking modes could be due to a fast time-scale $\left(\sim \tau_{1}\right)$ instability of the helically symmetric state attas.ad in the two-dimensional simulations. 17 Such an instability could play a role during the rapid quenching of the discharge. It has been previously shown that two-dimensional equilibria are unstable to symmetry preserving instabilities which tend to reduce the number of islands; i.e., Iead to coalescence. 20-21 This coalescence instability seems to require a threshold island thickness for onset. 20 It is therefore possible that, during the evolution of the tearing modes, which may be considered a succession of MHD equilibria because of the disparity in time scales, the island width may pass through a critical value at which a rapidly growing symmetry-breaking instability onsets. The nonlinear evolution of such an instability could lead to additiona: resistive reconnection and destruction of magnetic surraces, again on an intermediate time scale.

Our contribution is the calculation of the stability of single helicity nonlinear tearing mode states to MHD verturbations with allowance for variation of the perturbation in the helical symmetry direction of the island "equilibrium." The stability is analyzed by numerically solving the linearized equations as an initial value problem. This technique allows us to study the growth rate and mode structure of the most 
unstable mode. This mode will dominate the perturbation amplitude after a sufficiently long time if the initial conditions are not orthogonal to it. In solving the initial value problem rather than the corresponding eigenvalue problem, we have, however, given up information about the less rapidly growing and stable modes.

We have limited our explicit calculation to the stability of $m=2, n=1$ islands, which emanate from the $q=2$ surface, to perturbations of different helicity. These islands can achieve large widths for realistic current profiles; furthermore, once the island width exceeds the tearing layer width, the evolution occurs on the resistive time scale. 22 The poloidal flux function $\psi$ then obeys, at each instant, the equilibrium equation

$$
\nabla^{2} \psi=J(\psi)-2
$$

with $J$ the axial current density. The concept of MHD stability of a sequence of such states is then a systematically valid one. If we define $\Omega^{\prime}$ as the wavevector of the perturbation in the ignorable direction [see Eq. (16)], we find that, for $\mathrm{n}^{\prime}=0$, the coalescence instability is recovered. As $n$ ' is increased, the growth rate decreases because the stabilizing energy required to bend the strong axial magnetic field is increased. We find that the mode becomes stable for $m=2, n=1$ islands if $n$ ' is made large enough $i^{\prime}=1 / 2$ ) to satisfy the axial periodicity 
requirements as applied to the cylindrical model of the small inverse aspect ratio torus. We conclude that, for an $m=2$, $n=1$ island equilibrium, excitation of other helicities in the three-dimensional numerical calculations ${ }^{18}$ may be related to nonideal or nonlinear effects.

In Section II cine equations and ordering scheme are presented and the linearized equations are derived. The numerical results and discussion are cuntained in section III.

\section{EQUATIONS}

The results in this section have been previously derived by strauss. 23 We present them here for completeness and clarity. We begin by applying the small inverse aspect ratio tokamak ordering

$$
E-\frac{I_{\perp}}{I_{z}} \sim \frac{B_{\perp}}{B_{z}}-\frac{p^{l / 2}}{B_{z}} \ll 1
$$

to the MHD equilibrium equations

$$
\begin{aligned}
& D=\stackrel{J}{\check{C}} \times \underline{B}-\nabla p \\
& \nabla \times \underset{\sim}{\mathrm{B}}=\frac{4 \pi}{C} \mathrm{~J} .
\end{aligned}
$$


Here $L_{L}$ and $L_{z}$ are characteristic dimensions for variation perpendicular to and along the axial direction, respectively, $B_{1}$ and $B_{z}$ are the corresponding magnetic field components and p is the pressure. From Eq. (2) we find that the variation $\Delta B_{z} \sim \varepsilon^{2} B_{z}$. It then follows from Eq. (3) that $J_{*} \sim \varepsilon$ and $J_{1} \sim \varepsilon^{2}$. Taking the curl of Eq. (2) and using these results we Find that, to lowest order,

$$
\underset{\mathrm{B}}{\mathrm{B}} \nabla \mathrm{J}_{\mathrm{z}}=0
$$

We now assume a helical symmetry direction and introduce the independent variables

$$
\begin{aligned}
& r=\left(x^{2}+y^{2}\right)^{1 / 2} \\
& \tau=\theta-\frac{n}{\bar{m}} \frac{z}{R}
\end{aligned}
$$

2

where $r ; \theta, z$ are the usual cylindrical coordinates. Here $R \sim I_{z}$ is the "major radius" of the cylindrical tokamak and the ratio $\mathrm{n} / \mathrm{m}$ determines the helical pitch. For equilibria which are independent of $z$, we may introduce the magnetic flux function $\psi$ through the definition ${ }^{6}$ 


$$
\underset{\sim}{\mathrm{B}}=\underset{\sim}{\mathbf{z}} \times \nabla \psi+\frac{r \eta}{\mathrm{R}_{\mathrm{r}}} \mathrm{B}_{\mathbf{z}} \hat{\hat{\theta}}+\mathrm{B}_{\mathbf{z}} \hat{z}
$$

so that

$$
\underline{\mathrm{B}} \cdot \nabla \Psi=0
$$

The carets denote unit vectors. Equations (4) and (7) taken together imply that

$$
J_{z}=J_{z}(\psi)
$$

and substitution of (6) into Ampere's law (3) yields the equilibrium equation

$$
\nabla_{\perp}^{2} \psi=\frac{4 \pi}{c} J(\psi)-\frac{2 n}{m R} B_{z}
$$

Small perturbations with velocity $\underset{\sim}{\vee}$ about solutions of Eq. (9) satisfy the linearized equations

$$
n_{0} M \frac{\partial V}{\partial t}=-\nabla_{D}+\frac{1}{C}\left(\underset{\sim}{J} \times \underset{\sim O}{B_{0}}+\underset{J_{0}}{J} \times \underset{\sim}{B}\right)
$$

$$
=-\nabla\left(p+\frac{\underset{B}{B} \cdot \underset{B}{*}}{4 \pi}\right)+\frac{1}{4 \pi}(B_{0} \cdot \nabla_{\sim}^{B}+\underset{\sim}{B} \cdot \underbrace{B}_{0}),
$$




$$
\begin{aligned}
& \frac{\partial \underline{B}}{\partial t}=\nabla \times\left(\underline{v} \times B_{0}\right) \\
& =\left({\underset{\sim}{\mathrm{B}}}_{\mathrm{O}} \cdot \nabla\right) \underset{\sim}{\mathrm{V}}-{\underset{\sim}{\mathrm{B}}}_{\mathrm{O}} \nabla \cdot \underset{\sim}{\mathrm{V}}-(\underline{v} \cdot \nabla){\underset{\sim}{\mathrm{B}}}_{\mathrm{O}}, \\
& \frac{\partial p}{\partial t}+\alpha p_{o} \nabla \cdot v+\underline{v} \cdot \nabla p_{o}=0 .
\end{aligned}
$$

Here equilibrium quantities have a zero subscript, $M$ is the ion mass, and $x$ is the adiabatic index. In order for the large restoring pressure force $\nabla_{B} \cdot O_{\sim} / 4 \pi$ to be balanced by the potentially destabilizing terms $\underset{\sim}{\mathrm{B}} \cdot \overrightarrow{\mathrm{O}^{\mathrm{B}}}+\underset{\sim}{\mathrm{B}} \cdot \underset{\sim}{\mathrm{B}} \mathrm{O}^{\prime}$, the perturbation must satisfy $p \cdot v \sim \varepsilon^{2} v_{1} / a$. Comparison of perpendicular and $z$ components of the momentum equation $(\partial / \partial z-\varepsilon / a)$ yield the additional estinate $V_{z} \sim \varepsilon V_{\mathcal{L}}$. These two estirites allow the introduction of a stream function

$$
\underline{v}=\hat{z} \times \nabla \phi
$$

to the reguired order. Introduction of (II) into (10b) shows that we may express the perturbed magnetic field in terms of a flux function $\psi$ through

$$
\underset{\sim}{B}=\underline{\mathbf{z}} \times \nabla \psi
$$


which satisfies

$$
\frac{\partial \psi}{\partial t}=\hat{z} \times \nabla \psi_{0} \cdot \nabla \phi+B \hat{z} \tilde{z} \cdot \nabla \phi
$$

Here, as elsewhere, the operation $\hat{z} \cdot \nabla$ is understood to be performed with $\tau$ fixed.

Performing the operation $\bar{z} \cdot \nabla \times$ on Eq. (10a) and assuming constant density $\mathrm{n}_{\mathrm{c}}$, we obtain the equation

$$
\frac{\partial}{\partial t} \nabla_{\perp}^{2} \phi=\frac{1}{4 \pi n_{0} M}\left[\hat{z} \times \nabla \psi \cdot \nabla \nabla_{1}^{2} \psi_{0}+\hat{z} \times \nabla \psi_{0} \cdot \nabla \nabla_{\perp}^{2} \psi+B_{z}(\hat{z} \cdot v) \nabla_{\perp}^{2} \psi\right]
$$

for the evolution of the stream function. Numerical solutions of Eqs. (13) and (14) for particular solutions $\psi_{0}$ of F.q. (9) are presented in the next section.

\section{NUMERICAL RESULTS AND DISCUSSION}

The equilibriun functions $\psi_{0}(I, \tau)$ were obtained from the nonlinear tearing mode two-dimensional mass code. ${ }^{7}$ The $m=2$ islands grew from initial perturbations of "flat" current profiles w. ich corresponded to initial safety factor profiles of the form

$$
g(x)=c\left[I+\left(x / r_{0}\right)^{8}\right]^{1 / 4} .
$$


Flat current profiles were chcsen to insure large island widths since a threshold width for instability was expected. The initial $q=2$ surface was located at $r=0.7 I_{\max }$ and the values of $c$ and $r_{0}$ were adjusted to yield $g(0)$ of 1.4 and 1.1 . Since the results are similar: for the two values of $q(0)$, we present here only those for $q(0)=1.1$. Contours of the flux function $\psi_{a}$ for an island width $W \sim 0.4 r_{\max }$ are shown in Fig. 1. Since $\partial \psi_{0} / \partial z=0$, solutions of Eqs. (13) and (14) were zought of the form

$$
\left(\begin{array}{l}
\phi \\
\psi
\end{array}\right)(\underset{\sim}{r}, t)-\exp \left(i n^{\prime} z / R\right)\left(\begin{array}{l}
\phi \\
\psi
\end{array}\right)(r, \tau, t)
$$

and the time behavior was studied for several values of $n$ ". The linearized equations were advanced via a leapfrog scheme. Fourier analysis in $\tau$ was used to invert the Iaplace operator which appears on the left-hand side of Eq. (14). Equation (13) for $\psi$ was solved directly in $(r, i)$ space. Typically 50 evenly spaced radial grid points and $32 \tau$ grid points were employed.

An attempt was made to choose an initial perturbation which would efficiently excite the coalescence instability. This instability acts to reduce the number of islands by convecting flux within the islands towards one $x$ point and away from the other. The return flow occurs exterior to the islands. For $m=2$ islands, the principal $t$ dependence of the 
perturbation is of the form exp $( \pm i \tau)$. The $T$ dependence of $\psi_{0}$ couples al1 odd $t$ modes $\exp \left(\operatorname{im}^{\prime} \tau\right), m^{\prime}= \pm 1, \pm 3, \pm 5, \ldots$ Because of the finite resolution of the numerical difference scheme, we can hope to accurately represent only those modes $m^{\prime}<N_{T} / 2$ where $N_{\tau}$ is the number of grid points used to represent the $i$ variable. Fortunately, previous results for the coalescence instability show that the amplitude of harmonics decreises rapidly as $\mid m$ ' | increases, 20 enabling us to accurately represent the most unstable mode with an achievable value for $\mathrm{N}_{T}$. We choose the initial conditions $y=0$,

$$
{ }_{ \pm m}+ \pm i r\left|m^{\prime}\right|\left(r-r_{x}\right)\left(r-r_{\text {max }}\right)
$$

with $r_{x}$ the radius of the island $x$ points which were at $\tau=0$, $\tau$. When $n$ ' was small enough for instability to occur, excitation

with any odd value of $\mathrm{m}$ ' resulted in growth of the mode.

After a few growth times, the amplitude of the most unstable mode overwhelms that of the other modes and the spatial structures of $\phi$ and $\psi$ become time independent. Figures (2a) and (?b) show, respectively, the level contours of $\phi$ and $\psi$ for $n^{\prime}=0$ at time $t=18 t_{0}$, where $t_{0} \equiv \rho^{1 / 2} \mathrm{Rm}_{\mathrm{m}} /\left(\mathrm{B}_{\mathrm{z}} \mathrm{n}\right)$. For these parameters, the level contours show no perceptible change after time $t=12 t_{0}^{-1}$, whereupon the mode amplitudes take on exponential time behavior, with growth rate $y=0.31 t_{0}^{-1}$. The growth rate monotonically 
decreases with increasing $n^{\prime}$ as show: in Fig. 3. For an $m=2$ equilibrium configuration, and for m' odd, n' must be a halfinteger in order to fulfill the periodicity requirements in a large aspect ratio torus. Neither of the equilibria studied $[q(0)=1.1,1.4]$ showed any evidence of instability when $n^{\prime}=0.5$, even for large island widths $w=0.4 r_{\max }$. In conclusion, we have demonstrated that for an $m=2$, $n=1$ island equilibrium, excitation of other helicities which satisfy the periodicity requirements in a small inverse aspect ratio torus is not related to an ideai MHD instability. The observed excitation of these helicities in the three-dimensional numerical calculations may thus be related to effects ignored in our calculations such as: nonideal terms in the Ohm's law, nonlinear effects, or time dependence or the $m=2$ island. It is noteworthy, however, that the minimum n' needed to satisfy the periodicity conditions in a torus is given by $n^{\prime}=1 / m, m$ being the azimuthal mode number of the equilıbrium island. Therefore, our results do not preclude the possibility of an idial MHD instability of differing helicity in the case of a higher m island equilibrium. 


\section{ACKNOWLEDGMENTS}

We gratefully acknowledge useful diszussions regarding numerical questions with Drs. S. C. Jardin and J. Manickam.

This work was supported by United states Department of Energy Contract No. EY-76-C-02-3073, and also by United Stater Air Force office of Scientific Research Contract No. F $44620-75-\mathrm{C}-0037$. 
REFERENCES

${ }^{1}$ S. Von Goeler, W. Stodiek, and N. Sauthoff, Phys. Rev. Lett. 33,1201 (1974).

${ }^{2}$ s. $V$. Mirnov and I. B. Seminov, in Plasma Physics and Contralled Nuclear Fusion Research 1976, Vol. I, 301 (International Atomic Energy Agency, Vienna, 1977).

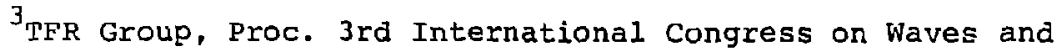
Instabilities in Plasmas, Palaisseau, France, 1977.

${ }^{4}$ N. R. Sauthoff, S. Von Goeler, and W. Stadiek, Princeton University Plasma Physics Laboratory Report PPPL-1379, January 1978.

${ }^{5}$ H. P. Furth, J. Killeen, and M. N. Rosenbluth, Phys. Fluids $\underline{6}, 459$ (1963).

${ }^{6}$ R. B. White, D. A. Monticello, , N. Rosen'lluth, and B. V. Waddell, Phys. Fluids 20, 800 (1977).

${ }^{7}$ B. V. Waddell, D. A. Monticello, M. N. Rosenbluth, and R. B. White, Nucl. Fusion 16, 528 (1976).

${ }^{8}$ D. Biskamp and H. Welter in Plasma Physics and Controlled Nuclear Fusion Research 1976, Vol. I, 579 (International Atomic Energy Agency, Vienna, 1977).

${ }^{9}$ M. N. Rosenbluth, D. A. Monticello, H. R. Strauss, and R. B. White, Phys. Fluids I9, 1987 (1976). 
${ }^{10}$ R. B. White, D. A. Monticello, and M. N. Rosenbluth, Phys. Rev. Lett. 39, 1618 (1977).

${ }^{1 l_{A}}$. B. Rechester and M. N. Rosenbluth, Phys. Rev. Lett. 40, $38(1978)$.

$12 \mathrm{~J}$. A. Krommes, R. G. Kleva, and C. Oberman, Princeton University Plasma Physics Laboratory Report PPPI-1389, May, 1978 submitted to

13 J. D. Callen, Phys. Rev. Lett. 39, 1540 (1977).

${ }^{14} \mathrm{~J}$. M. Finn, Nucl. Fusion 15, 845 (1975).

${ }^{15}$ J. M. Finn, Phys. Fluids 20, 1749 (1977).

16 T. H. Stix, Phys. Rev. Lett. 30,833 (1973); A. B. Rechester and T. H. Stix, Phys. Rev. Lett. 36, 587 (1976).

${ }^{17}$ E. J. Valeo and P. K. Kaw, Bull. Am. Phys. Soc. 22, 1172 (1977).

${ }^{18}$ H. R. Hicks, B. Carreras, J. A. Holmes, and B. V. Waddell, Oak Ridge National Laboratory Report ORNL/TM-6096 (1978).

${ }^{19}$ B. Carreras, B. V. Waddell, and H. R. Hicks, Oak Ridge National Iaboratory Report ORNL/TM-6175, March, 1978.

20 J. M. Finn and P. K. Kaw, Phys. Fluids 20, 72 (1977).

${ }^{21}$ D. Biskamp and $\mathrm{K}$. Schindler, Plasma Phys. 13, 1013 (1971).

22p. H. Rutherford, Phys. Fluids 16, 1903 (1973).

${ }^{23}$ H. R. Strauss, phys. Fluids 19, 134 (1976). 


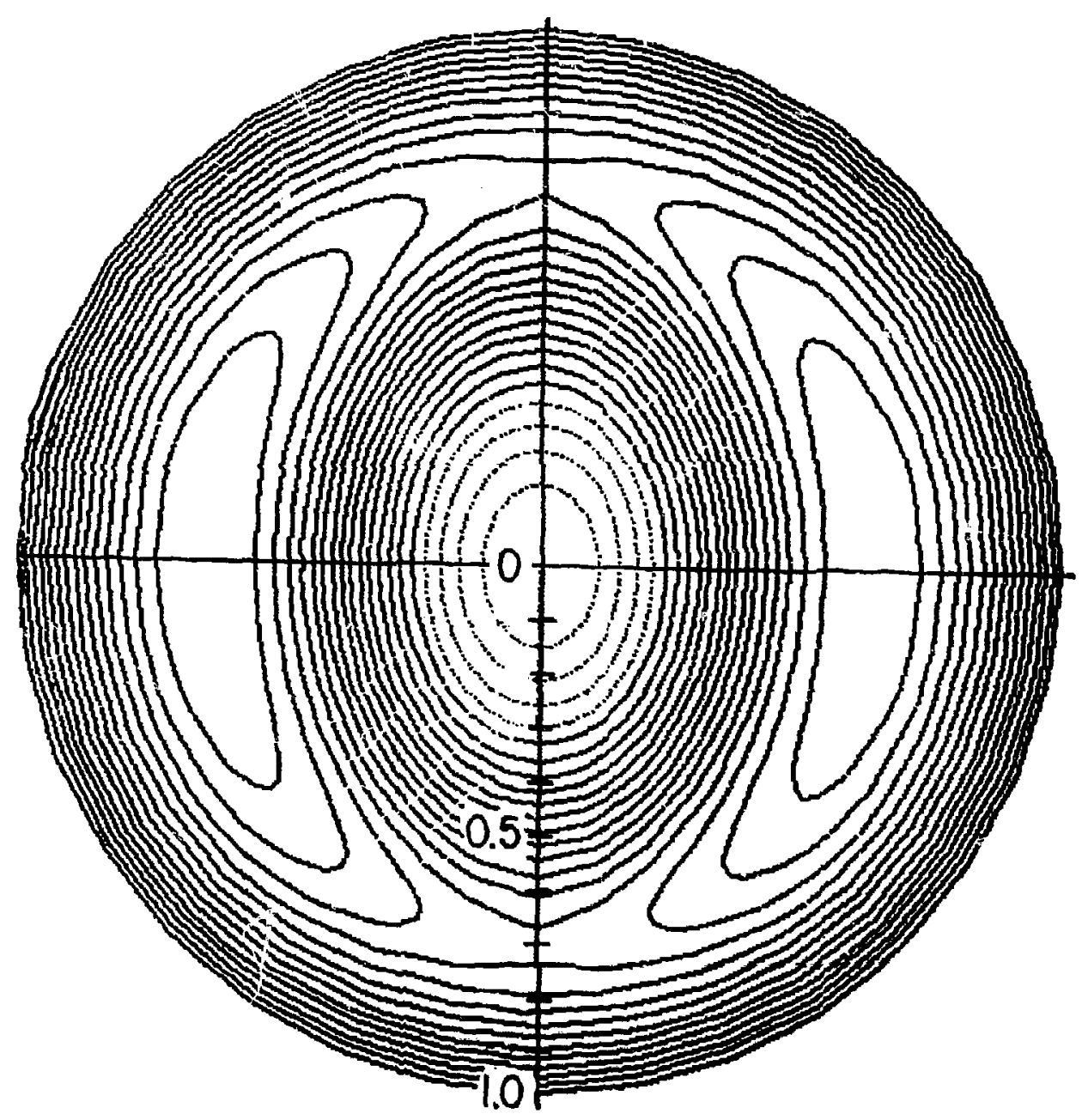

Fig. 1. Helically symmetric equilibrium flux surfaces $\psi_{0}$ takeñ from MASS code for a run with a "flat" initial current profile and an on-axis safety factor $q(0)=1.1$. 

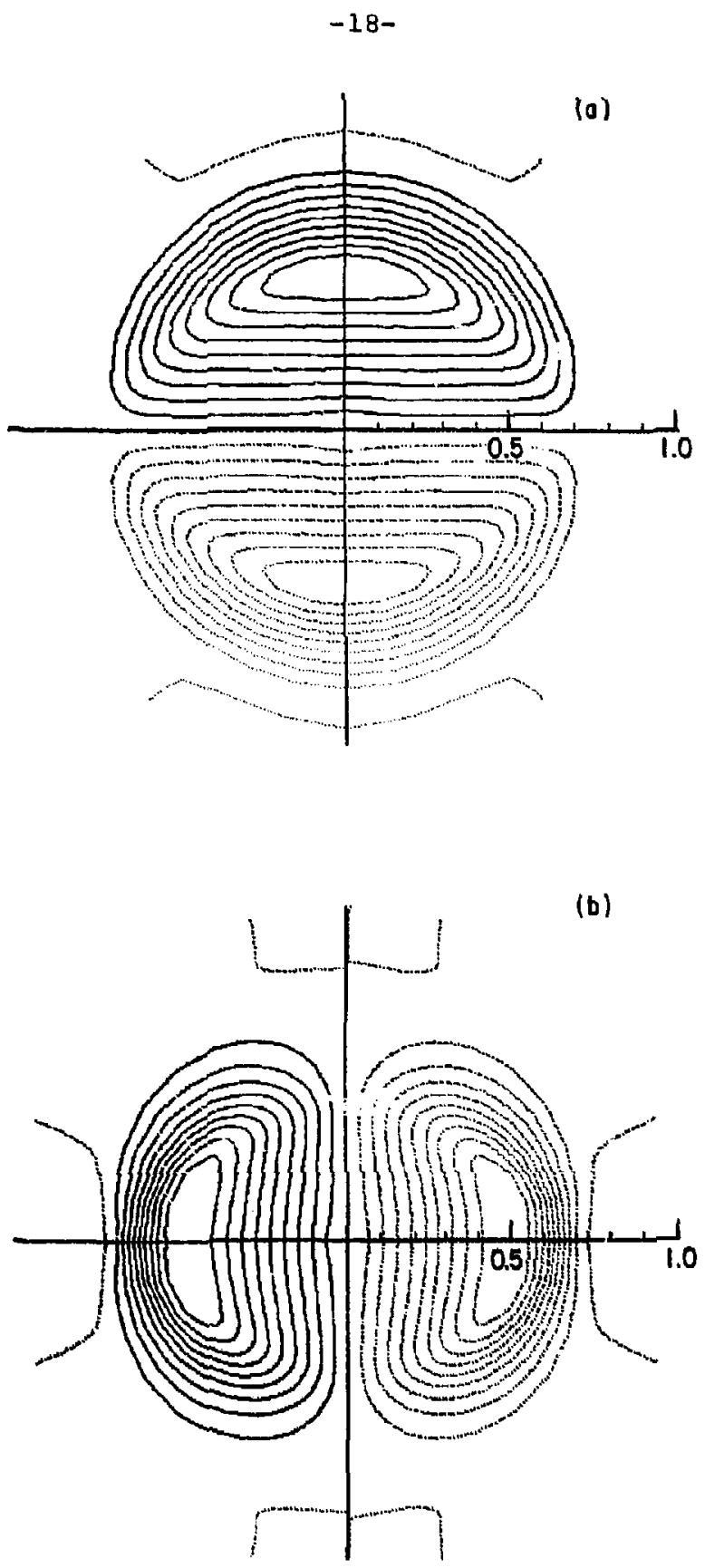

782260

Fig. 2. Contours of constant amplitude of the perturbation velocity potential $\phi(a)$ and vector potential $\psi(b)$ at time $t / t_{0}=18$, for $n^{\prime}=0$. 


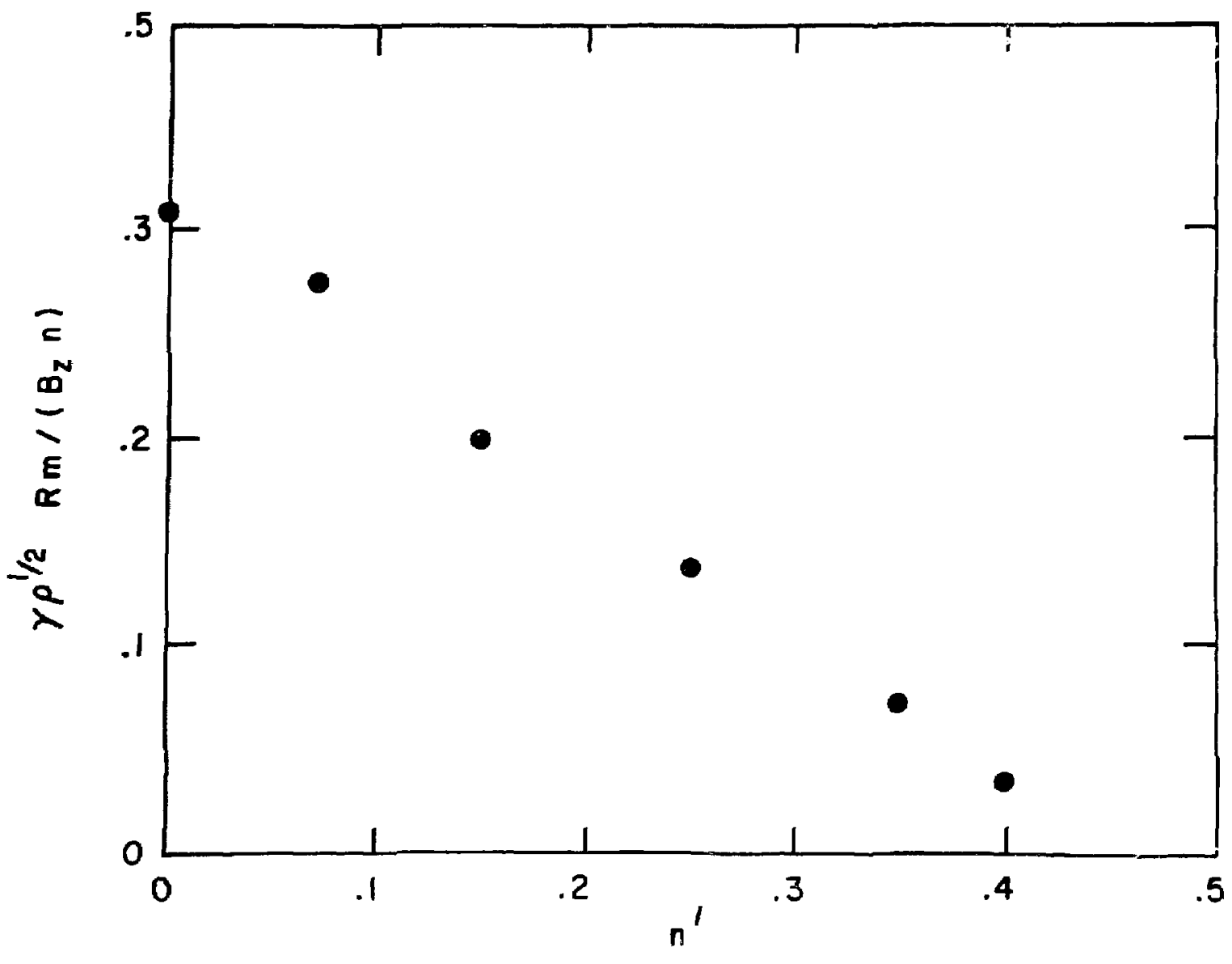

782261

Fig. 3. Growth rate vs $n^{\prime}$. 